PROCEEDINGS OF THE

AMERICAN MATHEMATICAL SOCIETY

Volume 128, Number 4, Pages 1197-1201

S 0002-9939(99)05203-X

Article electronically published on August 3, 1999

\title{
A CHARACTERIZATION OF MÖBIUS TRANSFORMATIONS
}

\author{
ROLAND HÖFER \\ (Communicated by Christopher Croke)
}

\begin{abstract}
Let $n \geq 2$ be an integer and let $\mathcal{D}$ be a domain of $\mathbb{R}^{n}$. Let $f: \mathcal{D} \rightarrow \mathbb{R}^{n}$ be an injective mapping which takes hyperspheres whose interior is contained in $\mathcal{D}$ to hyperspheres in $\mathbb{R}^{n}$. Then $f$ is the restriction of a Möbius transformation.
\end{abstract}

\section{InTRODUCTION}

Let $n \geq 2$ be an integer. A theorem of A.D. Alexandrov [1] states that any bijective transformation of $\mathbb{R}^{n+1}$ which preserves the Lorentz distance 0 between pairs of points in both directions is the product of a Lorentz transformation and a dilatation. The following Theorem 1.3 is due to A.D. Alexandrov [2], J.A. Lester 7], and I. Popovici and D.C. Rădulescu [9] and generalizes Alexandrov's theorem.

Definition 1.1. Let $n \in \mathbb{N}, n \geq 2$. For $x, y \in \mathbb{R}^{n}$ let $x \cdot y$ denote the standard euclidean product between $x$ and $y$. The Lorentz product, resp. Lorentz distance, between $x, y \in \mathbb{R}^{n+1}$ is defined by

$$
\begin{aligned}
x \diamond y & :=x_{1} y_{1}+\ldots+x_{n} y_{n}-x_{n+1} y_{n+1}, \\
d(x, y) & :=(y-x) \diamond(y-x) .
\end{aligned}
$$

Definition 1.2 (cf. [6] ). Let $n \in \mathbb{N}, n \geq 2$.

a) Let $\mathcal{D} \subset \mathbb{R}^{n}$. A mapping $f: \mathcal{D} \rightarrow \mathbb{R}^{n}$ is the restriction of a Möbius transformation if $\mathbb{R} \sigma_{1}(f(x))=\mathbb{R}\left(\sigma_{1}(x) A_{1}\right)$ is satisfied for all $x \in \mathcal{D}$, where

$$
\sigma_{1}(z):=\left(\frac{1-z \cdot z}{2}, z, \frac{1+z \cdot z}{2}\right)
$$

for all $z \in \mathbb{R}^{n}$, and where $A_{1}$ is an $(n+2) \times(n+2)$-Lorentz matrix, $A_{1} M_{1} A_{1}^{T}=$ $M_{1}:=\operatorname{diag}(1, \ldots, 1,-1)$.

b) Let $\mathcal{D} \subset \mathbb{R}^{n+1}$. A mapping $f: \mathcal{D} \rightarrow \mathbb{R}^{n+1}$ is the restriction of a Lie transformation if $\mathbb{R} \sigma_{2}(f(x))=\mathbb{R}\left(\sigma_{2}(x) A_{2}\right)$ for all $x \in \mathcal{D}$, where

$$
\sigma_{2}(z):=\left(\frac{1-z \diamond z}{2}, z, \frac{1+z \diamond z}{2}\right)
$$

for all $z \in \mathbb{R}^{n+1}$, and where $A_{2}$ is an $(n+3) \times(n+3)$-matrix with $A_{2} M_{2} A_{2}^{T}=$ $M_{2}:=\operatorname{diag}(1, \ldots, 1,-1,-1)$.

Received by the editors June 4, 1998.

1991 Mathematics Subject Classification. Primary 51B10; Secondary 51M04, 51M09.

Key words and phrases. Möbius transformation, Lie transformation, mappings preserving hyperspheres, Alexandrov's theorem for domains. 
Theorem 1.3. Let $\mathcal{D}$ be a domain (i.e. an open, connected subset) of $\mathbb{R}^{n+1}, n \geq 2$. Let $f: \mathcal{D} \rightarrow \mathbb{R}^{n+1}$ be a mapping such that

$$
d(x, y)=0 \quad \Leftrightarrow \quad d(f(x), f(y))=0
$$

for all $x, y \in \mathcal{D}$. Then $f$ is the restriction of a Lie transformation.

Alexandrov's theorem and Theorem 1.3 are important results in a modern field of geometrical research which is called characterizations of geometrical mappings under mild hypotheses [3, 4, [8]. In particular no regularity assumptions such as differentiability or even continuity are needed in these kinds of characterizations. In the same sense, C. Carathéodory proved [5 that any injective mapping of a domain $\mathcal{D}$ of $\mathbb{R}^{2}$ to $\mathbb{R}^{2}$ is the restriction of a Möbius transformation if the following condition is satisfied:

The image of any circle contained with its interior in $\mathcal{D}$, is itself a circle.

\section{Results}

There is a close connection between Carathéodory's theorem and Theorem 1.3 $(n=2)$. In fact we will generalize Carathéodory's theorem to arbitrary dimensions with the help of Theorem 1.3 .

Theorem 2.1. Let $n \geq 2$ be an integer and let $\mathcal{D}$ be a domain of $\mathbb{R}^{n}$. Let $f: \mathcal{D} \rightarrow$ $\mathbb{R}^{n}$ be an injective mapping such that $f(H)$ is a hypersphere, whenever $H \subset \mathcal{D}$ is a hypersphere and the interior of $H$ is contained in $\mathcal{D}$. Then $f$ is the restriction of a Möbius transformation.

Definition 2.2. A similarity of $\mathbb{R}^{n}, n \geq 2$, is a mapping $f: \mathbb{R}^{n} \rightarrow \mathbb{R}^{n}, f(x)=$ $k x Q+t$ where $k>0, t \in \mathbb{R}^{n}$, and $Q$ is an orthogonal $n \times n$-matrix, $Q Q^{T}=E$.

It is well known that a mapping $f: \mathbb{R}^{n} \rightarrow \mathbb{R}^{n}$ which is induced by a Möbius transformation is a similarity. Hence, Theorem 2.1 implies the following corollary.

Corollary 2.3. Let $n \geq 2$. Let $f: \mathbb{R}^{n} \rightarrow \mathbb{R}^{n}$ be an injective mapping such that images of euclidean hyperspheres are euclidean hyperspheres. Then $f$ is a similarity.

Now let $\mathcal{D}$ be the set $I^{n}:=\left\{x \in \mathbb{R}^{n} \mid x \cdot x<1\right\}$ of points in Poincaré's sphere model of $n$-dimensional hyperbolic geometry, $n \geq 2$. A hyperbolic hypersphere in $I^{n}$ is a euclidean hypersphere which is contained in $I^{n}$. If $f: I^{n} \rightarrow I^{n}$ is induced by a Möbius transformation and if $f$ is surjective, then $f$ is a hyperbolic motion.

Corollary 2.4. Let $n \geq 2$. Let $f: I^{n} \rightarrow I^{n}$ be a bijection of $n$-dimensional hyperbolic space which maps hyperbolic hyperspheres onto hyperbolic hyperspheres. Then $f$ is a hyperbolic motion.

\section{Proof of Theorem 2.1}

We show that, whenever $H$ is a hypersphere contained in $\mathcal{D}$ such that the interior $I$ of $H$ is also contained in $\mathcal{D}$, then $\left.f\right|_{I}$ is the restriction of a Möbius transformation. This implies Theorem 2.1 since

a) Any Möbius transformation is uniquely determined by its restriction to any non-empty open subset of $\mathbb{R}^{n}$.

b) For any two points $x, y \in \mathcal{D}$, there is a finite sequence $I_{1}, \ldots, I_{k} \subset \mathcal{D}$ of interiors of hyperspheres with $x \in I_{1}, y \in I_{k}, I_{j} \cap I_{j+1} \neq \emptyset$ for all $j \in\{1, \ldots, k-1\}$.

Let $H$ be a hypersphere contained in $\mathcal{D}$ such that the interior $I$ of $H$ is also contained in $\mathcal{D}$. 
1. Let $I^{\prime}$ denote the interior of the hypersphere $H^{\prime}:=f(H)$. Then either $f(I) \subset I^{\prime}$ or $f(I) \subset \mathbb{R}^{n} \backslash\left(H^{\prime} \cup I^{\prime}\right)$.

Proof. Let $x, y \in I$. Then there is a hypersphere $H_{1} \subset I$ which contains $x$ and $y$. Since $f$ is injective and $f\left(H_{1}\right)$ is a hypersphere, either $f\left(H_{1}\right) \subset I^{\prime}$ or $f\left(H_{1}\right) \subset$ $\mathbb{R}^{n} \backslash\left(H^{\prime} \cup I^{\prime}\right)$. Thus $f(x), f(y)$ are on the same side of $f\left(H_{1}\right)$.

2. Let $\mu: \mathbb{R}^{n} \backslash I^{\prime} \rightarrow \mathbb{R}^{n}$ denote the restriction of a Möbius transformation which satisfies $\mu\left(H^{\prime}\right)=H^{\prime}$ and $\mu\left(\mathbb{R}^{n} \backslash\left(I^{\prime} \cup H^{\prime}\right)\right) \subset I^{\prime}$. Let $g: H \cup I \rightarrow \mathbb{R}^{n}$ be defined by $g:=\left.f\right|_{H \cup I}$ if $f(I) \subset I^{\prime}$, and $g:=\left.\mu \circ f\right|_{H \cup I}$ if $f(I) \subset \mathbb{R}^{n} \backslash\left(H^{\prime} \cup I^{\prime}\right)$. Then $g(I) \subset I^{\prime}$, $g(H)=H^{\prime}$, and $g$ is an injective mapping which takes hyperspheres in $H \cup I$ to hyperspheres in $H^{\prime} \cup I^{\prime}$.

3. Let $H_{1} \subset I$ be a hypersphere with interior $I_{1}$. Then $g\left(I_{1}\right)$ is contained in the interior $I_{1}^{\prime}$ of $H_{1}^{\prime}:=g\left(H_{1}\right)$, and $g\left(I \backslash\left(H_{1} \cup I_{1}\right)\right)$ is contained in the exterior of $H_{1}^{\prime}$.

Proof. Let $z \in I \backslash\left(H_{1} \cup I_{1}\right)$. There is a hypersphere $H_{2} \subset H \cup I$ with $z \in H_{2}$, $\#\left(H \cap H_{2}\right)=1$ and $H_{1} \cap H_{2}=\emptyset$. Then $H_{2}^{\prime}:=g\left(H_{2}\right) \subset H^{\prime} \cup I^{\prime}, g(z) \in H_{2}^{\prime}$, $\#\left(H^{\prime} \cap H_{2}^{\prime}\right)=1$ and $H_{1}^{\prime} \cap H_{2}^{\prime}=\emptyset$. Hence $g(z) \notin I_{1}^{\prime}$, and $g\left(I \backslash\left(H_{1} \cup I_{1}\right)\right) \subset$ $I^{\prime} \backslash\left(H_{1}^{\prime} \cup I_{1}^{\prime}\right)$. From the proof of 1 . we know that $g\left(I_{1}\right)$ is either contained in the interior or in the exterior of $H_{1}^{\prime}$. We take a hypersphere $H_{3} \subset I$, \# $\left(H_{1} \cap H_{3}\right)>1$. Then $H_{3} \cap I_{1} \neq \emptyset$ and $\#\left(H_{1}^{\prime} \cap g\left(H_{3}\right)\right)>1$. Hence $g\left(H_{3} \cap I_{1}\right) \cap I_{1}^{\prime} \neq \emptyset$ and $g\left(I_{1}\right) \subset I_{1}^{\prime}$.

Definition 3.1. Two hyperspheres $H_{1}, H_{2} \subset \mathbb{R}^{n}$ are in interior (exterior) contact if $\#\left(H_{1} \cap H_{2}\right)=1$ and $H_{i}$ is contained in the interior (exterior) of $H_{j}$ where $(i, j)=(1,2)$ or $(i, j)=(2,1)$.

4. Two hyperspheres $H_{1}, H_{2} \subset I$ are in interior (exterior) contact iff $g\left(H_{1}\right), g\left(H_{2}\right)$ are in interior (exterior) contact.

Proof. Since $g$ is injective, $\#\left(H_{1} \cap H_{2}\right)=1$ iff $\#\left(g\left(H_{1}\right) \cap g\left(H_{2}\right)\right)=1$. The assertion now follows from 3 .

Definition 3.2. For any hypersphere $H_{1}$ let $\gamma\left(H_{1}\right) \in \mathbb{R}^{n}, \rho\left(H_{1}\right)>0$ denote the euclidean center and radius of $H_{1}$. Let $\lambda\left(H_{1}\right):=\left(\gamma\left(H_{1}\right), \rho\left(H_{1}\right)\right) \in \mathbb{R}^{n} \times \mathbb{R}_{>0}$.

5. Two distinct hyperspheres $H_{1}, H_{2}$ of $\mathbb{R}^{n}$ are in interior contact iff the Lorentz distance between $\lambda\left(H_{1}\right)$ and $\lambda\left(H_{2}\right)$ is zero.

6. The set $\mathcal{C}:=\left\{\lambda\left(H_{1}\right) \mid H_{1} \subset I\right.$ is a hypersphere $\}$ is a domain of $\mathbb{R}^{n+1}$.

Proof. $\mathcal{C}=\left\{x \in \mathbb{R}^{n} \times\right] 0, \rho(H)[\mid d(x, \lambda(H))<0\}$ is open and connected.

7. The mapping $\varphi:=\lambda \circ g \circ \lambda^{-1}: \mathcal{C} \rightarrow \mathcal{C}^{\prime}:=\left\{\lambda\left(g\left(H_{1}\right)\right) \mid H_{1} \subset I\right.$ is a hypersphere $\}$ satisfies $d(x, y)=0$ iff $d(\varphi(x), \varphi(y))=0$ for all $x, y \in \mathcal{C}$.

Proof. From 5. and 4., for all distinct hyperspheres $H_{1}, H_{2} \subset I$,

$$
\begin{aligned}
d\left(\lambda\left(H_{1}\right), \lambda\left(H_{2}\right)\right)=0 & \Leftrightarrow H_{1} \text { and } H_{2} \text { are in interior contact } \\
& \Leftrightarrow g\left(H_{1}\right) \text { and } g\left(H_{2}\right) \text { are in interior contact } \\
& \Leftrightarrow d\left(\lambda\left(g\left(H_{1}\right)\right), \lambda\left(g\left(H_{2}\right)\right)\right)=0 .
\end{aligned}
$$


8. From 7. and Theorem [1.3, $\varphi$ is the restriction of a Lie transformation, i.e. there is an $(n+3) \times(n+3)$-matrix $A_{2}=:\left(a_{i j}\right)_{i, j=1, \ldots, n+3}$ as in Definition $\left.1.2 \mathrm{~b}\right)$, such that $\mathbb{R} \sigma_{2}(y)=\mathbb{R}\left(\sigma_{2}(x) A_{2}\right)$ for all $x \in \mathcal{C}, y=\varphi(x)$.

Definition 3.3. A light line of $\mathbb{R}^{n+1}$ is a line $u+\mathbb{R} v, u, v \in \mathbb{R}^{n+1}, v \neq 0$, where $d(v, v)=0$.

9. $\left.f\right|_{I}$ is the restriction of a Möbius transformation.

Proof. Let $x \in I$. Let $l_{1}, l_{2}$ be two distinct light lines which contain $(x, 0)$. Then $\{(x, 0)\}=l_{1} \cap l_{2} \subset \partial \mathcal{C}$. The images $\varphi\left(l_{i} \cap \mathcal{C}\right) \neq \emptyset$ are contained in uniquely determined light lines $l_{i}^{\prime}, i=1,2$. Since $\varphi$ is continuous, $\{(g(x), 0)\}=l_{1}^{\prime} \cap l_{2}^{\prime}$ is contained in $\partial \mathcal{C}^{\prime}$. Hence for all $x \in I$, we have $\mathbb{R} \sigma_{2}((g(x), 0))=\mathbb{R}\left(\sigma_{2}((x, 0)) A_{2}\right)$ which implies

$$
\begin{gathered}
\mathbb{R} \sigma_{1}(g(x))=\mathbb{R}\left(\sigma_{1}(x) A_{1}\right), \\
\sigma_{1}(x) \cdot\left(a_{1, n+2}, \ldots, a_{n+1, n+2}, a_{n+3, n+2}\right)=0
\end{gathered}
$$

where $A_{1}$ is the $(n+2) \times(n+2)$-matrix obtained from $A_{2}$ by deleting the $(n+2)$ th row and $(n+2)$ th column. Equation (3.2) is a quadratic equation in $x=\left(x_{1}, \ldots, x_{n}\right)$ which holds for any $x \in I$, and we obtain $a_{1, n+2}=\ldots=a_{n+1, n+2}=a_{n+3, n+2}=0$. Together with $A_{2} M_{2} A_{2}^{T}=M_{2}$ we have $A_{1} M_{1} A_{1}^{T}=M_{1}$, where $M_{1}$ is chosen as in Definition 1.2 a). Equation (3.1) implies that $g$ is the restriction of a Möbius transformation. Hence also $\left.f\right|_{I}$ is the restriction of a Möbius transformation.

Remark 3.4. It is possible to prove Theorem 2.1 by Carathéodory's theorem. If $n=3$ and $f: \mathcal{D} \rightarrow \mathbb{R}^{3}$ is injective and has the sphere preserving property, then we can apply Carathéodory's theorem to any hypersphere $H \subset \mathcal{D}$ whose interior is contained in $\mathcal{D}$, after removing a point $p \in H$ and $f(p) \in f(H)$, to show that $f$ is a Möbius transformation between $H$ and its image $f(H)$. This Möbius transformation is the restriction of the same Möbius transformation for all hyperspheres. Induction proves the result for all $n \geq 2$.

I would like to thank the referee for helpful comments, especially Remark 3.4

\section{REFERENCES}

1. A. D. Alexandrov. Seminar report. Uspekhi Mat. Nauk, 37(3):187, 1950.

2. A. D. Alexandrov. On the axioms of relativity theory. Vestnik Leningrad Univ. Math., 19:5-28, 1976.

3. W. Benz. Characterizations of geometrical mappings under mild hypotheses: Über ein modernes Forschungsgebiet der Geometrie. Hamb. Beitr. Wiss.gesch., 15:393-409, 1994.

4. W. Benz. Real Geometries. BI Wissenschaftsverlag, Mannheim, Leipzig, Wien, Zürich, 1994. MR 95k:51019

5. C. Carathéodory. The most general transformations of plane regions which transform circles into circles. Bull. Am. Math. Soc., 43:573-579, 1937.

6. T. E. Cecil. Lie Sphere Geometry. Springer-Verlag, New York Berlin Heidelberg, 1992. MR 94m:53076

7. J. A. Lester. A physical characterization of conformal transformations of Minkowski spacetime. Ann. Discrete Math., 18:567-574, 1983. MR 84g:83004b 
8. J. A. Lester. Distance preserving transformations. In F. Buekenhout, editor, Handbook of Incidence Geometry, pages 921-944, Amsterdam, 1995. Elsevier. MR 96j:51019]

9. I. Popovici and D. C. Rădulescu. Characterizing the conformality in a Minkowski space. Ann. Inst. H. Poincaré. Phys. Théor., 35:131-148, 1981. MR 83b:53011

Mathematisches Seminar, Universität Hamburg, Bundesstr. 55, 20146 Hamburg, GerMANY

E-mail address: hoefer@math.uni-hamburg.de 